\title{
Visibilidad y percepción en la construcción de un paisaje prehistórico. El caso de Calviá (Mallorca, Islas Baleares) ${ }^{1}$
}

\section{Visibility and Perception in the Building of a Prehistoric Landscape. The Case of Calviá (Mallorca, Balearic Islands)}

\author{
Alejandra Galmés Alba \\ Universidad Complutense de Madrid \\ alejandragalmes@gmail.com
}

Recibido: 11-01-2015

Aceptado: 09-04-2015

\begin{abstract}
RESUMEN
El presente trabajo se centra en el estudio del papel que juega el control visual del espacio en las prácticas sociales de las comunidades prehistóricas. Este trabajo se articula a partir de un estudio de caso, el término municipal de Calviá, situado en el sureste de la isla de Mallorca, para analizar las diferentes formas de monumentalidad arquitectónica y cómo estas se constituyen cómo un punto de referencia social dentro del paisaje.

Partiendo de una amplia horquilla temporal, que abarcaría el Bronce Naviforme (1550-850 AC), el periodo Talayótico (850-550 AC) y el Postalayótico (550-123 AC), se propone analizar los cambios y pervivencias en la construcción del paisaje, a través de estrategias de visibilidad, percepción y movimiento alrededor de los monumentos arquitectónicos. A través de la perspectiva de la Arqueología del Paisaje y mediante el uso de Sistemas de Información Geográfica (SIG) se propone un análisis de tendencias a largo plazo en la configuración social de un paisaje.
\end{abstract}

Palabras Clave: Arqueología del Paisaje, SIG, arquitectura monumental, Mallorca.

\begin{abstract}
The present paper aims to study the role that the visual control of the landscape plays in the social practises of prehistoric communities. Focusing on a case study in the South West of Mallorca, we seek to get a closer look into the changing patterns of monumentality and the role of monumental architecture to socially signify the landscape.

By embracing a long term perspective, including the Naviform Bronze Age (1550-850 AC), the Talayotic (850-550 AC) and the Postalayotic period (550-123 AC), we propose to analyze the changes and survivals in the construction of the landscape through strategies such as visibility, perception and movement around the monumental architecture. From a Landscape Archaeology perspective and with the use of GIS techniques, this paper aims to analyse long term perspectives in the construction of a social landscape.
\end{abstract}

KEY wORDs: Landscape Archaeology, GIS, Monumental Architecture, Mallorca. 


\section{Paisaje, monumentalidad y visibilidad. La construcción social de un paisaje}

El estudio que aquí presentamos pretende ser una introducción a las formas por las que se construye socialmente el paisaje, centrándonos fundamentalmente en dos variables: el control visual que se ejerce sobre el espacio y la monumentalidad arquitectónica como forma de semantizar dicho espacio. Así, el estudio de los patrones de visibilidad y la relación de estos con el movimiento a través del territorio son el punto de partida para intentar aproximarnos a la concepción social y política del paisaje de las comunidades que nos precedieron. Es decir, supone una forma de análisis para aproximarnos a cómo habitaron un lugar $\mathrm{y}$, especialmente, a cómo construyeron la red de relaciones que, a través del tiempo y del espacio daba sentido a este.

En la isla de Mallorca se han realizado algunos estudios de carácter espacial y territorial (Gili 1995; Rodríguez Alcalde 1995; Aramburu 1998; Pons Homar 1999; Salvà 2001) centrados especialmente en el estudio de patrones de asentamiento y de captación de recursos, aunque hay estudios que amplían esta perspectiva hacia una dimensión simbólica del paisaje (Calvo 2009). En este sentido, el presente trabajo intenta ser una aproximación al paisaje como un producto socio - cultural, es decir, supone un análisis de los esquemas de racionalidad que orientan y determinan la percepción y concepción del territorio por parte de las comunidades que lo habitan (Criado 1989). Esta idea, provoca la búsqueda de las estrategias que permitan interpretar el paisaje, no únicamente desde su materialidad, sino aunando esta con su dimensión ideal o imaginaria (Criado 1993:42). La idea de paisaje que aquí seguimos entiende que éste no es simplemente un espacio, una porción de tierra, sino que se convierte en paisaje en tanto que es experimentado por las personas. El espacio adquiere sentido al estar habitado, es decir, al tejerse a través de él una red de acciones, vivencias y memorias (Ingold 1993; 2011).

Una de las fórmulas por las que el ser humano da significado al espacio es a través de la arquitectura, ya que esta supone la creación deliberada de un espacio, haciéndolo tangible, visible y sensible (Tilley 1994). A diferencia de los espacios naturales, a los que se les pueden asignar significados sociales; la arquitectura necesita de la implicación humana para existir, lo que la con- vierte en un referente dentro de la experiencia y el sentido de un paisaje (Tilley 2004:78). En este sentido, la prehistoria de las islas Baleares se caracteriza por la monumentalidad de sus construcciones, que han sido un referente constante en el imaginario de las gentes. El calificativo de monumental suele hacer referencia a construcciones de grandes dimensiones y elaboración, sea cual sea su función o cronología (Trigger 1990). Sin embargo, el hecho de que nosotros consideremos que un edificio excede sus dimensiones prácticas supone estar juzgándolo desde un punto de vista actual. Por ello, creemos que la monumentalidad más bien debería ser definida como construcciones de grandes proporciones concebidas para resistir el paso del tiempo (Criado 1991:92); dicho de otro modo, supone que las comunidades humanas se hacen presentes en el espacio de un modo intencional, conspicuo y más o menos perdurable (Parcero 2005:13). Los monumentos son, por tanto, construcciones perdurables sobre las que se tejen todo un conjunto de prácticas que sirven como un repositorio de la memoria (Bradley 1993). La monumentalización de la arquitectura supone una voluntad consciente de exhibición, de visualización, mediante el espacio, de un modelo social y cultural (Criado 2012:279). Esta voluntad de visibilidad es la que nos lleva a preguntarnos qué papel juegan estos monumentos cómo referente y cómo lugar de significación del espacio. En este sentido, se debe tener en cuenta que el paisaje se configura a la vez como

an anonymous sculptural form always already fashioned by human agency, never completed, and constantly being added to, and the relationship between people and it is a constant dialectic and process of structuration: the landscape is both medium for and outcome of action and previous histories of action. Landscapes are experienced in practice, in life activities (Tilley 1994:23).

Esta experiencia cotidiana del paisaje hace que nunca pueda considerarse completo sino que se encuentra siempre en construcción (Ingold 1993:162). En este sentido, la ocupación de un espacio, de un territorio, no se construye únicamente a través de los patrones de asentamiento, entidades fijas en el espacio, sino también mediante la movilidad a través de dicho espacio (Parcero et al. 2009).

Este movimiento hace que, al final, el paisaje cuente, o más bien sea, una historia (Ingold 
1993:153); es una forma de generar una experiencia compartida, de explicar una narrativa, de fijar una comunidad. El paisaje acumula historias personales, colectivas, míticas, que son rememoradas a través del movimiento por él (Santos-Granero 2004). Se configura, así, como un espacio social cambiante construido a partir de la experiencia y la interacción humana (Tilley 1994:10). Así, se convierte en algo desordenado, complejo, caótico, dónde cada uno de sus componentes se entremezcla con el resto (Ingold 1993:54). Debemos, por tanto, encontrar un modo de trabajar con el desorden del paisaje, para entender cómo aquello que vemos en un momento determinado es una confluencia de acciones y de tiempos pasados que se entremezclan en un presente (González Ruibal 2006). Exponemos aquí una primera aproximación al análisis de la construcción social del paisaje y, a través de la arquitectura, a los modos de ordenar, concebir y percibir la red social articulada a través de este en la zona del suroeste de la isla de Mallorca.

\section{2. Área de estudio: la zona de Calviá (Mallorca)}

La delimitación de un ámbito para realizar un estudio paisajístico o territorial es siempre complicada, debido a la diferente percepción que tenemos del espacio en la actualidad, así como por la demarcación de unidades administrativas que poco tienen que ver con los límites naturales de los territorios. El presente trabajo se corresponde al actual municipio de Calviá, al suroeste de la isla de Mallorca. Aunque estamos condicionados por un límite actual, el área de Calviá presenta cierta coherencia geográfica conformando una pequeña península al sur del municipio delimitada por las últimas estribaciones de la Sierra de Tramuntana y por la línea de costa.

Podemos dividir el municipio en dos grandes zonas de características geográficas diferenciadas. Por un lado, la zona norte, en la que encontramos dos grandes valles cerrados por la Serra de Tramuntana, distribuidos paralelamente. Por otro lado, la zona sur, es decir, la península de Santa Ponça, cerrada al norte por las estribaciones montañosas y por el salobral de Palma Nova / Magaluf al este y la albufera de Santa Ponça al oeste; quedando el sur delimitado por una costa escarpada. Destaca en ella la zona de Santa Ponça, dónde encontramos una bahía que finaliza en una playa de 90 hectáreas, cerrada a ambos lados por formaciones montañosas (Albero et al. 2011a: 293-297). Al fondo de esta gran playa encontramos una planicie sedimentaria, lugar dónde se ubicó una albufera y marisma, que se habría extendido, desde esta, unos $200 \mathrm{~m}$ hasta el interior. Este lugar habría almacenado materiales sedimentarios aportados por el torrente del Galatzó y posiblemente fue un lugar de aguas estancadas (Camps y Vallespir 1998: 18; Esteban et al. 1991). Actualmente, tanto este salobral como el situado en la zona de Magaluf, se encuentran prácticamente desecados debido a la presión urbanística.

La hidrografía de la zona no presenta cursos de agua permanentes sino únicamente torrentes estacionales. Destaca el torrente del Galatzó, que nace al norte del valle homónimo, y al cual vierten la mayoría de torrentes de la zona, como el de Son Boronat, desembocando en la antigua albufera de Santa Ponça. Asimismo el torrente de Es Gorg vehicula el drenaje de la zona de Peguera (Albero et al. 2011a: 293-296). Sin embargo, se debe tener también en cuenta la presencia de algunas fuentes y manantiales naturales, así como el posible uso del agua de los acuíferos (Guerrero 1982: 13).

En cuanto a la vegetación de la zona, los datos antracológicos apuntan a que entre el 4000-2000 $\mathrm{BP}$, la principal formación vegetal de las Baleares era el ullastrar, basado principalmente en la presencia de Olea europaea, así como de Pinus halepensis, Pistacia lentiscus. Es decir, nos encontramos con una vegetación de carácter abierto, con un predominio de formaciones arbustivas, la denominada maquia mediterránea, aunque podría haber habido algunas formaciones de pinares, más o menos cerrados (Picornell 2012).

La selección de esta área de estudio, además de por el hecho de formar una zona geográficamente coherente, es debido a la importante investigación arqueológica que se ha llevado a cabo en este término municipal. Así, una de las ventajas que presenta el área de análisis es el elevado número de excavaciones arqueológicas realizadas en la zona. Estas intervenciones han permitido que actualmente contemos con multitud de datos sobre este zona, que van desde análisis cerámicos, paleobotánicos, arquitectónicos, faunísticos o metalúrgicos hasta interpretaciones sobre la economía y organización social de la zona (como ejemplos ver Guerrero et al. 2007; García Rosselló 2010; Albero 2011; Calvo y Aguareles (Ed.) 2011; Picornell 2012).

Para el presente estudio se han seleccionado un total de 70 yacimientos, clasificados según su 
tipología y adscripción cronológica. Siguiendo la variable temporal se han dividido los yacimientos analizados según su cronología: Bronce Naviforme Inicial (1600/1500-1300/1400 AC), Bronce Naviforme Final (1300/1400-900/850AC) (Albero et al. 2011b; Calvo y Guerrero 2011a; Calvo et al. 2011), Talayótico (850-650/550 AC) y Postalayótico (650-123 AC) (Guerrero et al. 2002; Calvo y Guerrero 2011b, c). La diferenciación entre el Bronce Naviforme Inicial y Final constata los cambios a nivel de gestión del territorio que supone la ocupación, en esta fase final, de lugares elevados, con control sobre la costa (Albero et al. 2011b; Calvo et al. 2011), como el Puig de Sa Morisca (hacia el 1300/1400 AC); $\mathrm{y}$ de lugares con un incipiente control visual del territorio, como el espacio después ocupado por el Turriforme de Son Ferrer (hacia el 1100 AC) o las laderas del Puig des Caragol (Albero et al. 2011a: 311-316)

En cuanto a la adscripción funcional de los yacimientos se ha seguido la realizada por el equipo de la Universitat de les Illes Balears en el monográfico sobre el patrimonio de este municipio (Calvo y Aguareles (Ed.) 2011). La adscripción funcional de los yacimientos se realizó estableciendo cuatro grandes grupos: yacimientos de hábitat, funerarios, rituales y estratégicos. En cuanto a la adscripción cronológica, en los casos posibles se llevó a cabo mediante la información proporcionada por excavaciones arqueológicas. En los casos en los que no ha sido posible, se acudió a datos de materiales en prospección en superfície. Asimismo, se usó el estudio del patrón de asentamiento para establecer la funcionalidad. Finalmente, se consideraron los paralelos en la tipología arquitectónica para determinar la posible funcionalidad y cronología del asentamiento. Sin embargo, esta última aproximación presenta problemas debido a la polifuncionaldiad que pueden presentar las estructuras arquitectónicas y los cambios de uso que pueden sufrir a lo largo del tiempo (Albero et al. 2011a: 298).

\section{Metodología}

La confección del presente trabajo se ha realizado utilizando esencialmente las herramientas que proporcionan los Sistemas de Información Geográfica (SIG). Las coordenadas espaciales de los yacimientos analizados han sido obtenidas mediante las fichas del Plan General de Ordenación Urbana (PGOU) del Ayuntamiento de Calviá. Los análisis se han realizado con un Modelo Digital del Terreno (MDT) de resolución $5 \times 5 \mathrm{~m}$ realizado por el Instituto Geográfico Nacional (IGN). Todos los análisis se han realizado mediante ArcGis 10.1.

El uso de Sistemas de Información Geográfica (SIG) resulta una herramienta útil para intentar aproximarnos a un momento concreto de un paisaje que hoy, evidentemente, está entremezclado con todo lo ocurrido a través de él hasta nuestros días. Aunque puede resultar una obviedad constatar que los paisajes cambian (Antrop 2005), quizás no lo es tanto en el caso de Calviá. El municipio de Calviá ha sufrido, desde los años 60 del siglo XX un importante impacto urbanístico, debido principalmente al desarrollo del turismo de masas, que supuso una rápida construcción de infraestructuras (Vives 2011). El área de estudio es un espacio mayoritariamente urbanizado en la actualidad lo que dificulta, si no imposibilita, la realización de estudios como éste únicamente partiendo de la experiencia en campo (fig. 1).

Se han realizado dos tipos fundamentales de análisis: mapas de visibilidad y Modelo de Acumulación del Desplazamiento Óptimo desde un origen (MADO). El uso de cuencas de visibilidad teórica (viewshed) (Weathley 1995; Llobera 2003) ha sido utilizado para observar cómo los monumentos arquitectónicos de los diferentes períodos analizados se relacionan con determinados patrones de visibilidad del territorio y cómo su visualización supone que se conviertan en marcadores y semantizadores del paisaje. Para calcular la visibilidad de los monumentos, se han

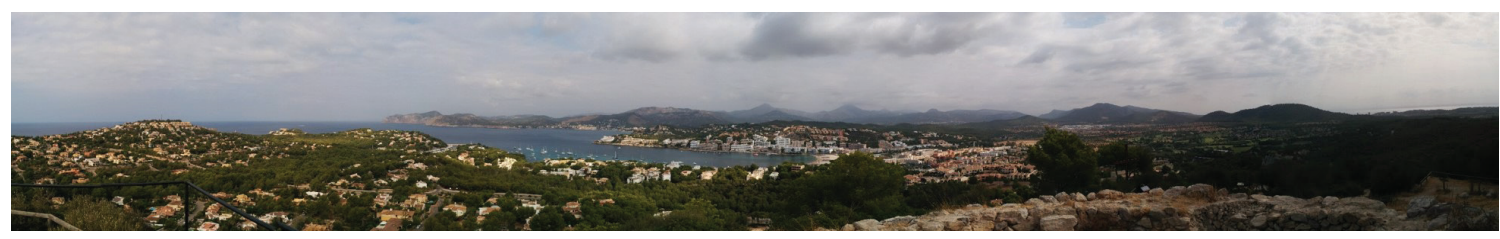

Fig. 1. Panorámica sobre la bahía de Santa Ponça y la antigua área de la albufera realizada desde el Puig de Sa Morisca. 
considerado unas alturas mínimas teóricas de las distintas tipologías constructivas, así como una altura mínima teórica del observador para calcular la visibilidad desde estas construcciones. Esta última, se ha establecido en $1.55 \mathrm{~m}$ basándonos en estudios de poblaciones de la Edad del Bronce, tanto en las Baleares como en casos comparativos (Rihuete 2000; Basildo et al. 2005).

Sin embargo, cabe mencionar que en la evaluación de los estudios de visibilidad se deben tener en cuenta los umbrales de visión del ojo humano. Para ello, se considera que el ojo humano puede percibir un objeto de $3 \mathrm{~m}$ de altura, con un ángulo de entre 1 y 5 minutos, a una distancia de entre 2 y $10 \mathrm{~km}$. Este rango varía dependiendo de las condiciones de visibilidad del ambiente, así como del contraste del objeto con los alrededores determinado por la vegetación, el color, etc. (Shang y Bishop 2000).

En segundo lugar, se han realizado estudios de patrones de movimiento en el intento de relacionar la influencia de factores físicos del movimiento con distintos elementos del registro arqueológico, tales como monumentos o asentamientos (Fábrega y Parcero 2007:121). En este sentido, se ha escogido la realización de un MADO o Modelo de Acumulación del Desplazamiento Óptimo desde un origen, que puede ser definido como

the representation of an accumulation model of lowest cost movement calculated from a given origin and without specific destination points (Fábrega - Álvarez 2006: 9).

Esta característica, es decir, la posibilidad de calcular el desplazamiento sin tener que condicionarlo mediante un punto de partida y un punto de llegada es, a nuestro entender, el mayor atractivo de este modelo. Metodológicamente, para su realización se tienen en cuenta tanto los factores físicos que condicionan el desplazamiento, esencialmente la fricción, es decir, la dificultad que supone el desplazarse en cada fracción de terreno; y el coste (Tobler 1993), o esfuerzo necesario ir de un punto a otro, teniendo en cuenta la distancia lineal y la fricción que los separa (Parcero - Oubiña et al. 2009). En la realización de este modelo se ha venido teniendo en cuenta tanto el terreno como la red hidrográfica para determinar la fricción y el coste. Sin embargo, en el presente caso, la hidrografía no se ha considerado un factor relevante que impida la movilidad ya que no contamos con cursos de agua permanentes y, además, está constatado el uso de la red de to- rrenteras como caminos en época histórica (Grimalt 2001).

\section{Hábitats para ser vistos: el bronce naviforme}

El Bronce Naviforme Inicial (1600/1500 1300/1400 AC) y el Bronce Naviforme Final (1400/1300-900/850 AC) se caracterizan por la construcción de los hábitats naviformes, llamados también navetas de habitación. Consisten en estructuras de hábitat de planta alargada en forma de herradura y un ábside más o menos apuntado, erigidas mediante técnica ciclópea. Los muros están construidos mediante una técnica de doble paramento, es decir, constan de una parte exterior realizada con grandes bloques de piedra y una interior, de bloques de menor tamaño, entre las cuales se coloca un relleno de piedras y tierra para dar consistencia a la construcción (Guerrero 2001; Garcia Amengual 2014). Estas edificaciones tienen unas dimensiones de unos 16-25 $\mathrm{m}$ de longitud por unos 5-7 $\mathrm{m}$ de anchura, y tendrían unos $3 \mathrm{~m}$ de altura, documentándose en algunas ocasiones altillos destinados al almacenaje. El techo estaría compuesto de una mezcla de ramaje, posiblemente acebuche, y un entramado vegetal impermeabilizado con arcilla, aunque se documentan algunos casos con cubiertas en piedra (Pons 1999:23-25; Calvo y Salvà 1999; Hernández et al. 2003; Salvà y Hernández 2009; Fornés et al. 2007; Garcia Amengual 2014).

La construcción del hábitat Naviforme supone una monumentalización del espacio doméstico. Se ha planteado que su forma característica podría estar relacionada con la tradición funeraria del momento anterior (1800-1550 AC), basada en hipogeos con corredor. La monumentalización del hábitat se podría relacionar con un uso intencional del prestigio de los antepasados. Además, se plantea que el uso de la técnica ciclópea se relacionaría con las fórmulas sociales usadas para evidenciar, retar y renegociar el orden social (Fornés et al. 2007: 325; Javaloyas et al. 2011; Albero et al. 2013). Así, estas edificaciones podrían haber funcionado como una fórmula de cohesión e identificación del grupo.

Los análisis de arqueología territorial (Gili 1995; Rodríguez Alcalde 1995; Pons 1999; Salvà 2001) han mostrado que los navetiformes se ubican en áreas con suelos fértiles, propicios para la agricultura, aunque también suelen contar a su alrededor con áreas de pastoreo u otros recur- 


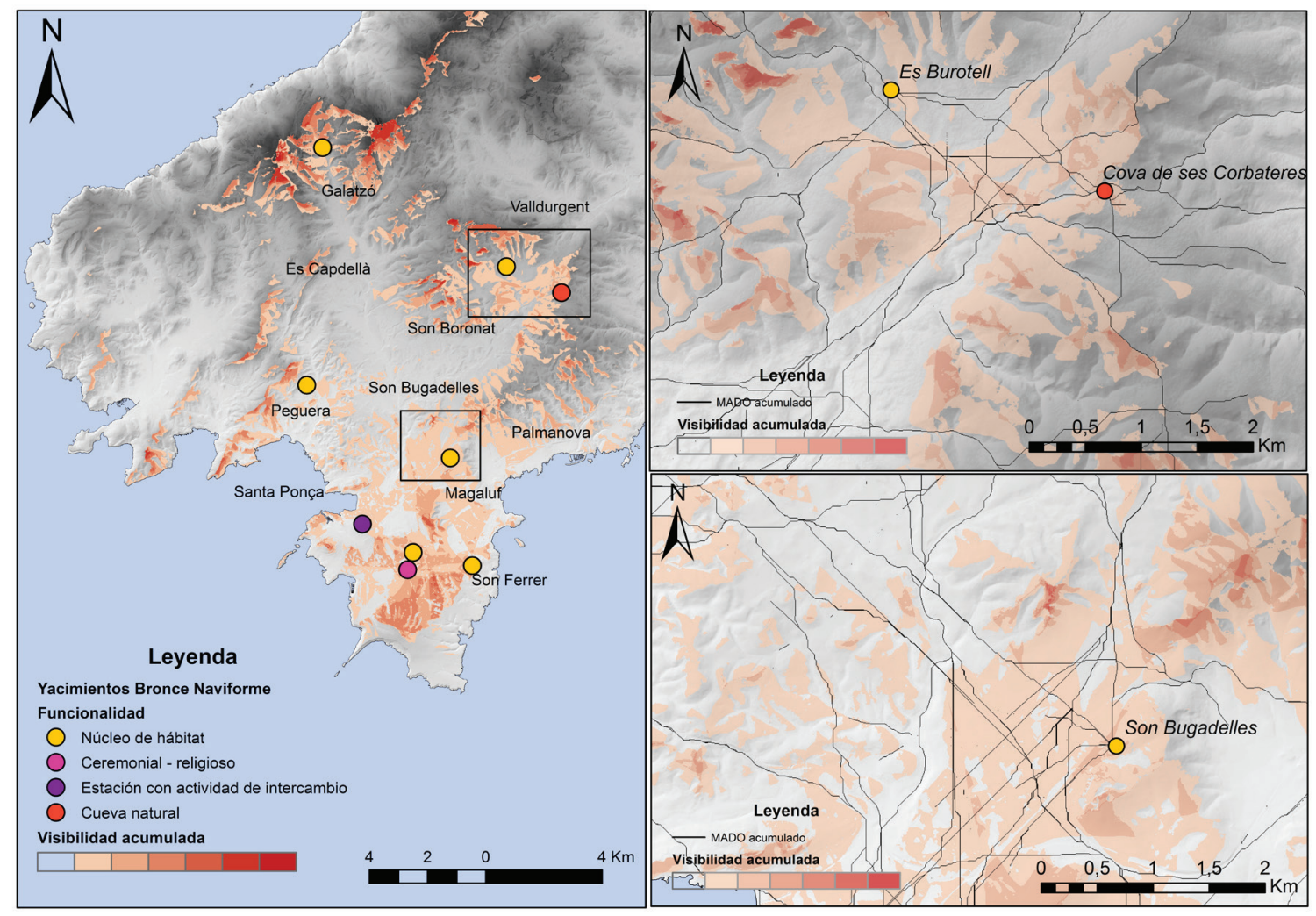

Fig. 2. Visibilidad acumulada general para el Bronce Final Naviforme y ejemplos de movilidad acumulada (MADO) en torno a estructuras naviformes.

sos, así como fuentes de agua. Se ha enfatizado el hecho de que los navetiformes se encuentren, generalmente, situados en lugares sin un valor estratégico o defensivo, ubicación que tampoco transmite diferencias en cuanto a jerarquía entre los asentamientos (Pons 1999:114).

En esta zona (fig. 2), los naviformes se localizan en los fondos de valle o en el llano, dónde se ubican las áreas potenciales de explotación agrícola. Los análisis de visibilidad muestran cómo desde estas edificaciones no parece que haya un control importante del territorio, sino que su localización podría haber tenido más relación con la explotación y significación del paisaje, dado que son edificios que, por su magnitud, habrían podido ser fáciles de ver y reconocer.

Esta hipótesis se refuerza con el análisis de movimiento (MADO) que muestra cómo los poblados naviformes tienden a concentrarse en áreas de acumulación de movimiento o en zonas de paso. Así, en el llano encontramos los naviformes de Son Ferrer, a unos $600 \mathrm{~m}$ de distancia de un hipogeo funerario (1800-1500 AC), sobre el que se construye algún tipo de edificación ha- cia el 1100-850 AC que se encuentra amortizada en el posterior conjunto talayótico (Calvo et al. 2005; 2014). Encontramos también en el llano de Son Ferrer el naviforme Alemany (Ensenyat 1971; Albero et al. 2013; Garcia Amengual 2014), una estructura actualmente aislada situada sobre una pendiente y a $700 \mathrm{~m}$ de la costa; presentando una amplia visibilidad sobre la zona del llano de Son Ferrer y situado en un área de alta movilidad. Al norte del municipio, encontramos estructuras naviformes en el paso que conecta el área de Valldurgent con el llano de Palmanova (el poblado de Es Burotell y la cueva funeraria de Ses Corbateres); y en la conexión de estas áreas con el llano interior de Santa Ponça (poblado de Son Bugadelles) (fig. 2). Por otro lado, en el fondo del valle del Galatzó encontramos el poblado de Ses Sínies, ubicado en un cerrado fondo de valle, con escasa visibilidad y cuya ubicación podría estar relacionada con actividades de pastoreo (Albero 2006). Si se conjugan los dos análisis, vemos cómo las líneas de movilidad son a su vez espacios visibles desde los yacimientos, aunque esta visibilidad se circunscribe a su perímetro 
más próximo. Vemos, por tanto, cómo los naviformes se ubican en áreas de tránsito, aunque no presentan una voluntad clara de controlar visualmente este posible movimiento.

Este esquema cambia durante el Bronce Final $(1400 / 1300-1100$ AC) momento en el que se produce una ocupación sin precedentes de la costa, especialmente de pequeños promontorios costeros (Salvà 2001; Salvà et al. 2002; Calvo et al. 2011). Esta ocupación coincide con la intensificación de los contactos, especialmente entre las islas de Mallorca y Menorca, lo que habría generado un habitus común, es decir, un conocimiento y unas prácticas compartidas en relación tanto a la cultura material (cerámica, metalurgia, arquitectura) como a prácticas simbólicas (Albero et al. 2011b). Dentro de esta estrategia de ocupación de la costa se deben enmarcar las primeras ocupaciones del Puig de Sa Morisca (120 m) (Guerrero et al. 2002; 2006a; 2007) hacia el $1300 \mathrm{AC}$; y la presencia de una estructura de tendencia naviforme en la ladera del Puig des Caragol (470 m) (Guerrero 1982:272; Albero 2006; Albero et al. 2011a:311-316). Ambas rompen con el patrón de asentamiento anterior y suponen el inicio de un mayor control visual del territorio que caracterizará al período Talayótico.

El hábitat naviforme se configura como una arquitectura monumental que semantiza el paisaje (Calvo 2009), construida para ser vista. La monumentalización del espacio de hábitat se relaciona con prácticas de cohesión e identificación del grupo y la visibilidad de estas construcciones desde los espacios circundantes, áreas de explotación agrícola, hacen que estas estructuras puedan haber funcionado como referentes visuales en el espacio. Asimismo, su ubicación en relación a las áreas de movilidad hace que fuesen edificaciones altamente visibles desde estas, lo que refuerza la idea de su papel como semantizador del paisaje.

\section{Hacia un paisaje visualmente controlado: el Talayótico}

Durante el período Talayótico (850-550 AC) la gestión de la arquitectura monumental cambia, pasando a realzar el espacio comunitario, con lo
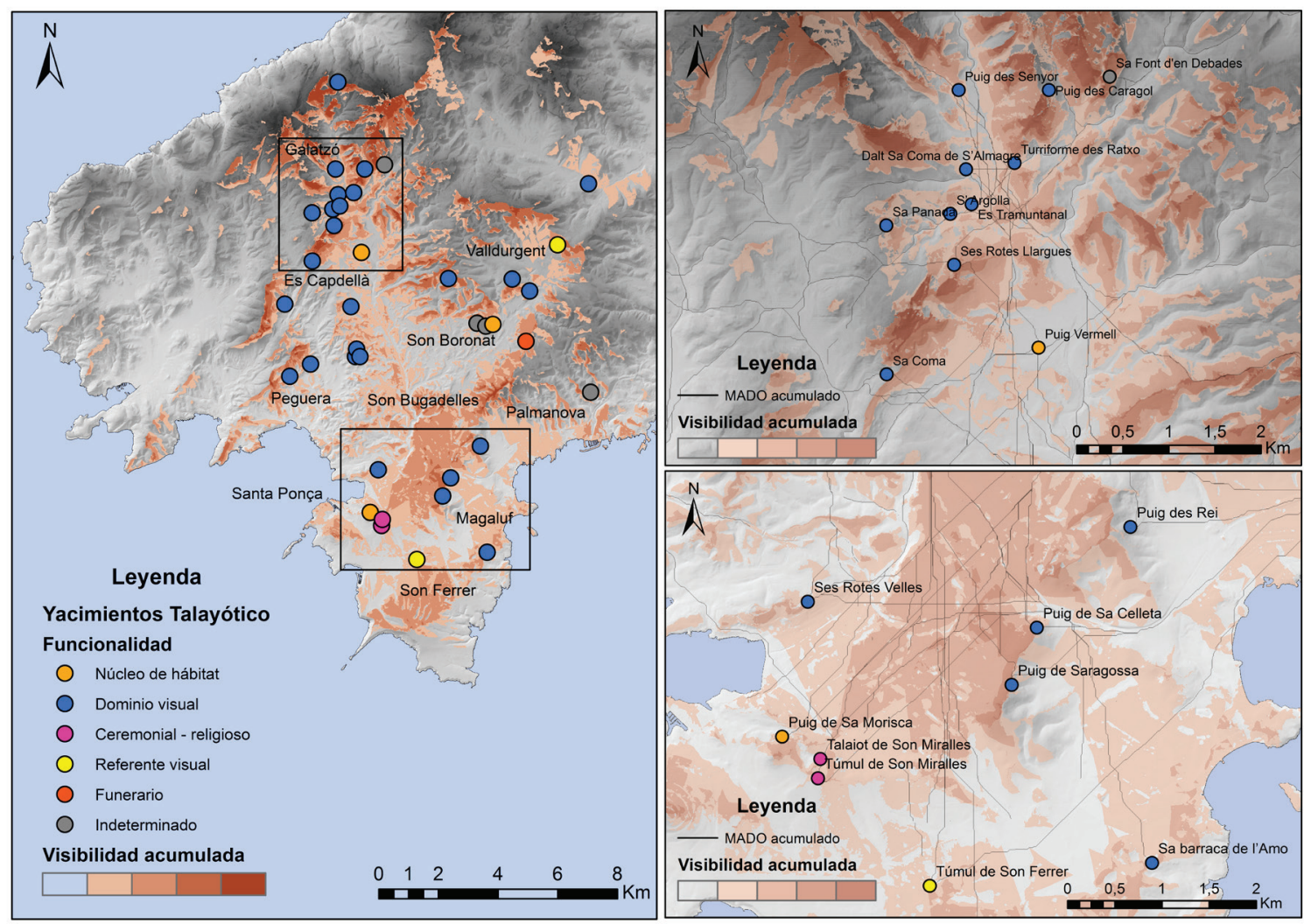

Fig. 3. Visibilidad acumulada general para el período Talayótico y ejemplos de movilidad acumulada (MADO). 

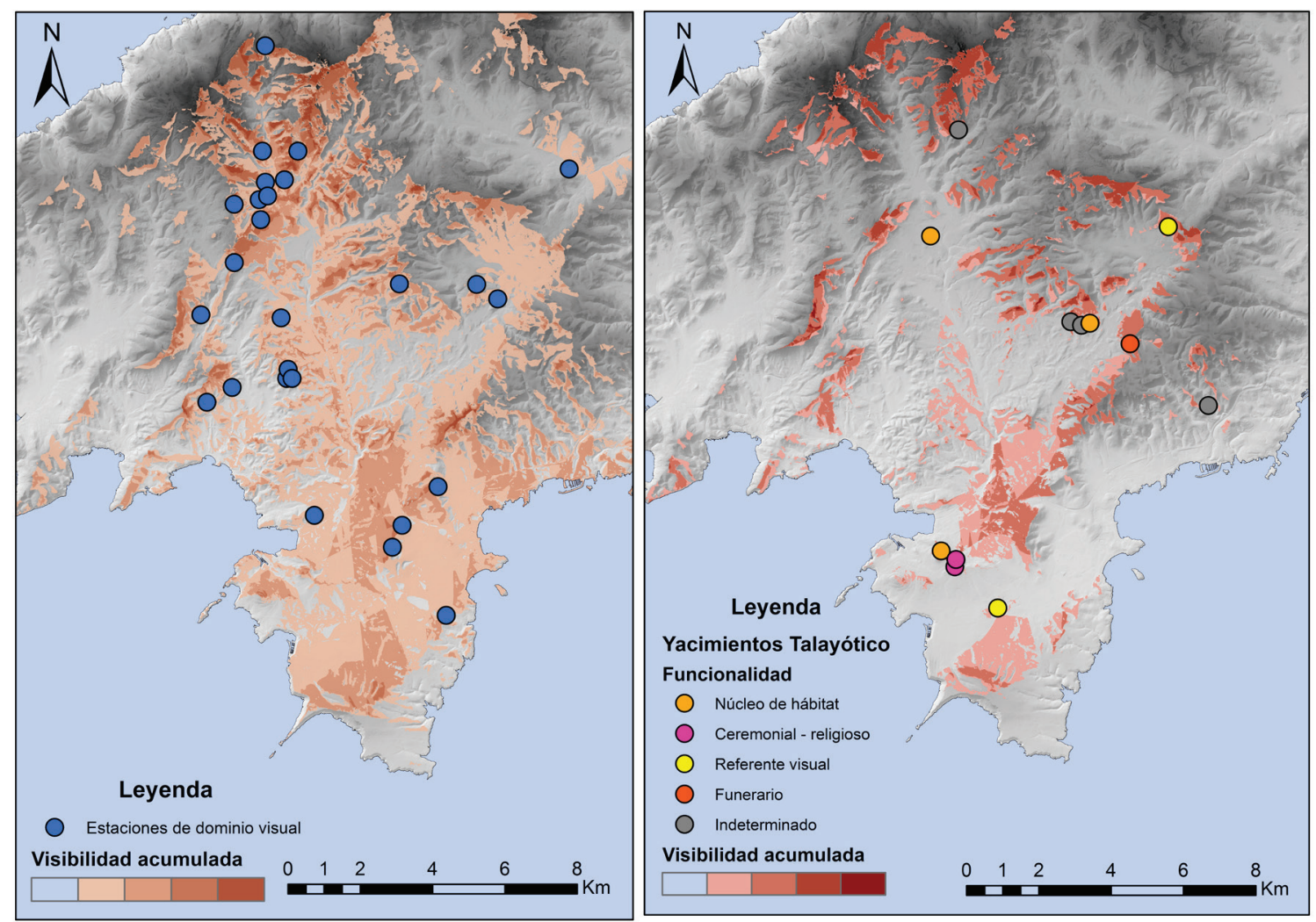

Fig. 4. Comparación entre la visibilidad acumulada desde las estaciones de dominio visual durante el período Talayótico y la visibilidad acumulada desde el resto de asentamientos.

que se visibilizan constructivamente espacios de gestión y reunión por encima de las estructuras de hábitat. Entre las fórmulas arquitectónicas monumentales de este período, la más conocida es el talayot, un espacio de reunión colectiva, con funciones de carácter económico, político y ceremonial; usándose sus azoteas para tareas de control del territorio (Lull et al. 2001:33-34).

El talayot es una fórmula arquitectónica que se define como un turriforme, construido mediante técnica ciclópea de doble paramento y con una planta de tendencia circular, aunque también se documentan algunos de planta cuadrada (Aramburu y López Quesada 1996). Normalmente, presentan una cámara interior, con o sin columna central, y tendrían una cubierta plana, de madera, preferentemente acebuche, ramaje y arcillas (Aramburu 1998; Guerrero 1999; Lull et al. 2001). Sin embargo, aunque esta es la estructura más conocida y estudiada, en estos momentos el espacio también se articula a través de otras construcciones de carácter comunitario como son los turriformes y las plataformas escalonadas. Este cambio de tendencia en la monumentalización de los espacios, del ámbito doméstico al edificio comunitario, es un proceso lento que se inicia durante el Bronce Final (c. 1000 AC) (Fornés et al. 2007).

El aumento de tipologías arquitectónicas monumentales (Aramburu 1998) supone una creciente antropización y semantización del paisaje que se ha relacionado con una reestructuración social y política de las comunidades isleñas debido a una alteración de los contactos con el exterior, caracterizados por una mayor presencia fenicia (Guerrero et al. 2002; 2007). Asimismo, esta mayor evidencia de las comunidades locales sobre el territorio mediante distintos hitos arquitectónicos que jalonan el territorio se ha relacionado con un auge demográfico (Aramburu 1998; Pons 1999; Calvo 2009). Se ha propuesto que este conjunto de factores podría haber conllevado una necesidad creciente de adscripción grupal al territorio, que se haría visible a través de distintos modelos arquitectónicos (Calvo 2009). Se propone así la construcción de un paisaje más cerrado, que giraría alrededor del 
poblado, dónde se concentra la población y alrededor del cual encontramos los distintos hitos arquitectónicos (Aramburu 1998; Calvo 2009). Los análisis de cuencas visuales (fig. 3) muestran como, en estos momentos, la ubicación de los asentamientos se relaciona con un mayor control visual del territorio. Durante este período se documenta una ocupación más intensa del espacio, tanto en el llano interior de Santa Ponça como en los valles. Esta mayor presencia sobre el territorio configura un espacio más cerrado y controlado visualmente. Este control visual, sin embargo, no se realiza desde los lugares de hábitat sino desde pequeños asentamientos, que se jalonan a lo largo de toda el área. Estas estaciones (fig. 4) forman una estrecha red que controla visualmente el espacio sin prácticamente solapamientos, siendo la mayor parte del territorio controlada desde uno o desde dos asentamientos. Dicho de otro modo, aproximadamente el $80 \%$ del territorio se visualiza desde uno o desde dos asentamientos, el 67\% desde tres y el $16 \%$ desde cuatro. Es decir, esta maraña de asentamientos supone la creación de una eficaz red de control visual del espacio.

Los análisis de movimiento (fig. 3), por su parte, muestran cómo estos asentamientos se localizan en áreas donde o bien hay una alta movilidad o bien cerca de puntos estratégicos de paso. Su número se intensifica en la zona de la Serra de Tramuntana, lo que podría estar relacionado con la multiplicidad de pequeños pasos y valles que comunican la zona montañosa con el interior de la península de Calviá. Por ejemplo, se localiza una alta concentración de ellos (Ses Rotes Llargues, Es Tramuntanal, Turriforme de Es Ratxo, S'Argolla, Puig des Senyor...) en el área que conecta los valles de Es Capdellà, Galatzó y el área de Andratx (fig. 3). En comparación, la visibilidad desde los núcleos de hábitat, localizados esencialmente en los fondos de valle y en el llano de Son Ferrer, se circunscribe a su área más próxima.

Vemos así cómo las edificaciones monumentales del período Talayótico se convierten en hitos desde los que se controla visualmente el paisaje pero, a su vez, son lugares de referencia dentro de él. Es decir, juegan un doble papel, ver y ser visto. La configuración de un espacio altamente controlado visualmente supone una mayor antropización y semantización del espacio. Es una fórmula mediante la que se construye un espacio más cerrado, más significado a través de arquitecturas que se relacionan con espacios y actividades de la comunidad.

\section{El control estratégico del espacio: el Postalayótico}

Durante el período Postalayótico (550-123 AC) el uso y función de la arquitectura monumental sufre un nuevo cambio. La creación de poblados de nueva planta sin presencia de arquitectura ciclópea, así como el abandono y amortización de algunos talayots muestran cómo estas edificaciones van perdiendo su poder de significación del territorio (Calvo 2009). Paralelamente, la creciente visibilidad del individuo frente al grupo en las prácticas funerarias (Hernández 1998) evidencia la transición hacia una sociedad cada vez más jerarquizada, puede que debido a una intensificación del contacto púnico ebusitano, especialmente a partir del siglo IV AC (Guerrero et al. 2002; Costa et al. 2004; Calvo y Guerrero 2011b; Hernández y Quintana 2013), con lo que también podría relacionarse el creciente número de yacimientos cercanos a la costa. Se plantea que en estos momentos la arquitectura monumental perdería buena parte de su carga simbólica como elemento de apropiación e identificación del paisaje ya que este proceso se realizaría a través de elementos económicos, sociales o ideológicos de otro tipo (Calvo 2009).

Los análisis de visibilidad muestran que los asentamientos activos en estos momentos se concentran preferentemente en los alrededores del Puig de Sa Morisca, lugar desde el que se centraliza el comercio con el exterior de la isla (Quintana 2000; Hernández y Quintana 2013). A su alrededor surgen distintas estaciones, relacionadas especialmente con actividades de hábitat y de producción, por lo que parece ser que tanto la población como las actividades artesanales se centralizan en esta área estratégica. La excavación de una de estas, el Turó de Ses Abelles (Camps y Vallespir 1998), muestra como la funcionalidad de este asentamiento se relaciona preferentemente con el procesado de materias primas, y pese a ser un espacio comunitario ha perdido ya el carácter monumental en su construcción.

La concentración de asentamientos (fig. 5) en los alrededores del Puig de Sa Morisca y, por tanto, conectados con el puerto natural de Sa Caleta, apunta a que el foco económico principal se traslada desde los terrenos agrícolas del interior hacia la costa, intensificándose en estos momentos los intercambios con el exterior. En general, se documenta un espacio mucho menos contro- 

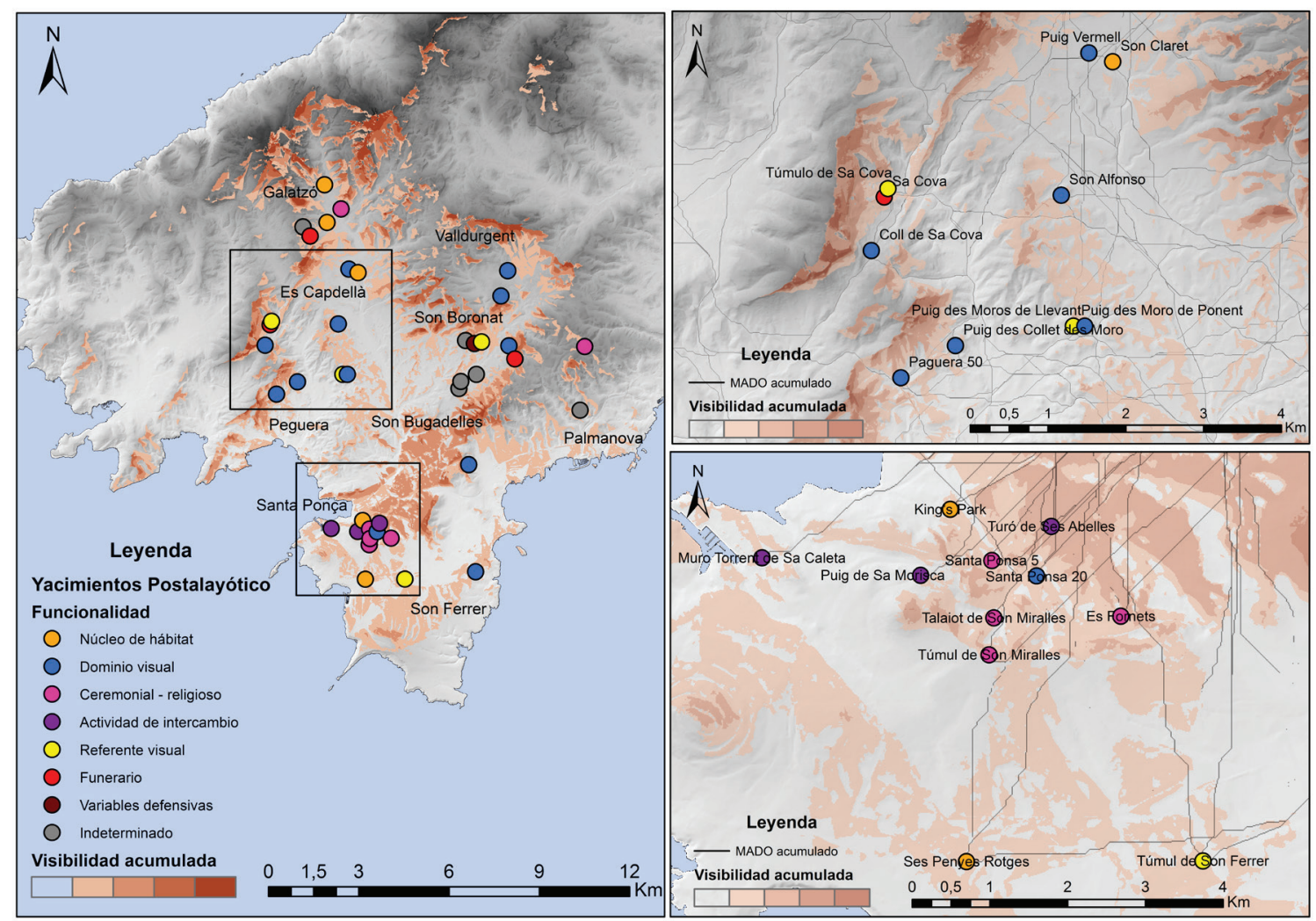

Fig. 5. Visibilidad acumulada general para el período Postalayótico y ejemplos de movilidad acumulada (MADO).

lado visualmente, que contrasta con una intensa y compacta red de asentamientos en esta zona, con una alta visibilidad entre ellos, lo que podría estar relacionado con el desarrollo de actividades sociales, económicas o políticas comunes. Estos nuevos asentamientos carecen de arquitectura de carácter monumental, lo que refuerza la idea de que ésta va perdiendo su papel semantizador del paisaje.

Por otro lado, surgen nuevos espacios de hábitat, sin presencia de arquitectura de carácter monumental. Entre ellos, destaca el poblado de Ses Penyes Rotges (Calvo 2002; Guerrero et al. 2006 b) (fig.5), al sur de la península de Santa Ponça, que hacia el siglo IV AC se convierte en un importante núcleo de población. Se aprecian, diferencias en la localización espacial del asentamiento, situado en una zona llana, con una visibilidad orientada fundamentalmente hacia el interior agrícola. Es decir, en este caso nos encontramos con un asentamiento de hábitat relacionado con la gestión de los recursos del interior, contrastando con el mayor papel del Puig de Sa
Morisca como referente visual hacia el exterior y vehiculador de los contactos extrainsulares.

Sin embargo, la arquitectura monumental, no pierde completamente su papel, sino que se reconfigura mediante nuevas fórmulas arquitectónicas, como los santuarios, edificaciones relacionadas con prácticas ideológicas o religiosas (Garcia Rosselló et al. 2000). Así, vemos como la monumentalidad deja de ser utilizada para la construcción de estructuras comunitarias, que cohesionaban y daban prestigio a una comunidad, y pasa a usarse en arquitecturas de carácter simbólico, reflejando un nuevo esquema de identidad y significación del espacio (Calvo 2009; Albero et al. 2011a: 357). En el área de estudio solamente se documenta una de estas estructuras, el santuario de Es Fornets (Guerrero 1982; Vallespir et al. 1987; Calvo 2002), situado a $1 \mathrm{~km}$ del poblado del Puig de Sa Morisca (fig. 5). Su localización hace que este yacimiento tenga cierta visibilidad sobre la antigua albufera de Santa Ponça y sobre el área norte del llano de Son Ferrer, y podría haber sido visible desde buena parte de los yaci- 
mientos que conforman la red alrededor del Puig de Sa Morisca.

Por otra parte, cabe mencionar en estos momentos la reutilización de espacios de épocas anteriores, como el hipogeo bajo el turriforme escalonado de Son Ferrer (Calvo et al. 2014) (fig. 5), que aproximadamente hacia el siglo VI -V AC vuelve a usarse como necrópolis de inhumación, enterrándose en él tanto adultos como, mayoritariamente, individuos infantiles (Garcias y Gloaguen 2003; Alesan y Malgosan 2005). Asimismo, se documentan también en esta estructura enterramientos perinatales en urnas de arenisca, ánforas recortadas y cerámicas de importación en la zona este del monumento, posiblemente con un significado simbólico (Calvo et al. 2014). La reutilización de este espacio otorga un nuevo significado a la construcción, que además de ser un marcador del paisaje, vuelve a tener el papel de necrópolis. El turriforme de Son Ferrer se configura como un lugar persistente en el paisaje (Schlanger 1992:97), teniendo ocupaciones sucesivas desde el Bronce Antiguo. Este yacimiento se sitúa en el centro del llano de Son Ferrer, y habría sido ampliamente visible desde buena parte de este espacio, lo que incide sobre su posible papel de referente simbólico del paisaje (Calvo et al. 2005).

Otro yacimiento que se configura como un lugar persistente es el Puig de Sa Morisca. Como veíamos, este lugar elevado presenta sus primeras ocupaciones durante el Bronce Final (c. 1400 AC) (Guerrero et al. 2002; 2006 a; 2007). Durante el Talayótico, este yacimiento se configura en dos ámbitos. Por un lado, un hábitat localizado en una vaguada, sin visibilidad sobre el entorno; y por otra, el conocido como castellum, una localizado sobre la elevación del Puig de Sa Morisca, a $201 \mathrm{~m}$ de altitud (Guerrero 1982:121; Vallespir et al. 1987; Calvo 2002). Aproximadamente hacia el siglo V AC (Guerrero et al. 2002) se configura un sistema de control del territorio sobre esta colina a través de cuatro torres, en un espacio que sirve como conector visual entre el interior de la península de Santa Ponça y la costa, controlando visualmente tanto la bahía y albufera de Santa Ponça como el puerto natural de Sa Caleta (fig. 5). Este lugar se configura como un lugar clave para entender las relaciones con el exterior de la isla, debido a que funciona como un lugar de contacto, documentándose especialmente materiales cerámicos provenientes de la colonia púnica de Ebussus (Guerrero 1997; 1999:97; Guerrero et al. 2002; Quintana 2000; Garcia Rosselló y Quintana 2003; Hernández y Quintana 2013). Las cuatro torres de la colina del Puig de Sa Morisca configuran un sistema que controla visualmente el área de santa Ponça, el llano interior de Son Ferrer, la costa de Magaluf y el área de Son Bugadelles.

En cuanto los análisis de movimiento (fig.5), en estos momentos vemos como la movilidad se concentra en áreas mucho más específicas, coincidiendo especialmente con la acumulación de asentamientos en los alrededores del Puig de Sa Morisca. Por otro lado, aunque, en general, el número de asentamientos destinados al control visual del territorio disminuye notablemente, seguimos encontrando yacimientos controlando las zonas de paso entre los distintos valles. Si bien el control visual general es menos intenso, se mantienen los puntos clave. Finalmente, cabe destacar que los yacimientos catalogados como estaciones de carácter ceremonial o religioso (tales como el santuario de Es Fornets o el Túmulo de Son Ferrer con sus reutilizaciones) (fig. 5) se ubican en áreas de acumulación del movimiento, por lo que podrían haber funcionado como espacios de referencia y semantización simbólica del espacio.

Vemos cómo, en estos momentos, el uso de la arquitectura monumental sufre un nuevo cambio, bien mediante nuevas fórmulas constructivas o bien mediante la reutilización y resignificación de espacios anteriores. En este periodo, la monumentalidad se asocia de especial forma con actividades de carácter simbólico - religioso, como en los casos del turriforme de Son Ferrer o del santuario de Es Fornets. En este período, el control visual del territorio se centra en áreas estratégicas, como el área de Santa Ponça, dónde se sitúa el Puig de Sa Morisca y la estrecha red de yacimientos que surgen en estos momentos a su alrededor.

\section{Conclusiones}

Este trabajo supone la continuación de estudios previos (Albero 2006; Calvo 2009; Albero et al. 2011 a) y supone un intento para comprender el papel cambiante de la arquitectura monumental, así como los patrones de asentamiento y los lugares que dan un significado social, cultural, simbólico y político a un paisaje. Un paisaje que no desaparece sino que es reinventado y permanentemente en construcción; es un ente dinámico y, 
a la vez, estratigráfico (Parcero 2000:15-16). Vemos como la arquitectura monumental es utilizada en cada momento en una categoría distinta del espacio social (el hábitat, el espacio comunal, el espacio simbólico), enfatizando el papel que tiene cada uno de estos en la configuración social y en la arena política de cada momento.

El uso de la arquitectura monumental enfatiza un espacio distinto en cada uno de los momentos analizados, convirtiendo a estos, y a las actividades que se asocian a ellos, como referentes en la experiencia del paisaje (Tilley 1994; 2004). Así, durante el Bronce Naviforme (1600/1500900/850 AC) vemos cómo los espacios domésticos, los naviformes, se convierten en ejes de referencia dentro del paisaje; unas estructuras hechas para ser vistas que conforman hitos dentro de sus áreas más inmediatas. Durante el Bronce Naviforme Final (1400/1300-900/850 AC) este esquema empieza a resquebrajarse, apuntando a un incipiente control del territorio que se consolidará en época Talayótica (850-650/550 AC). En estos momentos, la monumentalidad arquitectónica enfatiza espacios comunitarios, principalmente los talayots, centros de reunión de la comunidad y los turriformes, relacionados con actividades comunales y simbólicas y con el control estratégico del espacio. Se construye así un paisaje más cerrado y más controlado, cuyos hitos arquitectónicos reflejan a la comunidad que lo habita. El período Postalayótico (650-123 AC) supone a su vez un epílogo y una reestructuración de todo lo anterior. La monumentalidad arquitectónica deja de asociarse a los espacios cotidianos de la comunidad, restringiéndose su uso a edificaciones relacionadas con prácticas simbólico - religiosas, como los santuarios. Sin embargo, a la vez, se documenta la reutilización de espacios anteriores, como el turriforme de Son Ferrer, espacios que no dejarán de ser hitos de referencia dentro del paisaje. El control visual del territorio disminuye respecto al período anterior, aunque se mantiene en lugares estratégicos. En este sentido la acumulación de nuevos asentamientos en los alrededores del Puig de Sa Morisca enfatiza la importancia que adquiere esta zona como lugar clave de conexión con el exterior de la isla.

Al final, entendemos que un paisaje se constituye como un recuerdo y testimonio de las vidas y el trabajo de las generaciones que han pasado por él (Ingold 1993:152) y la arquitectura como una forma de unión de tiempo y espacio que supone la creación de lugares que se convierten en puntos fijos del paisaje en medio de la fluidez de la existencia (Richards 2000:544). El cambiante papel de la monumentalidad arquitectónica y su relación tanto con el control visual cómo con la significación del espacio a través del movimiento, permiten ver cómo esta enfatiza, hace visible (Criado 2012: 279) a la comunidad dentro del paisaje. Así, vemos como la arquitectura resalta distintos espacios en cada uno de los períodos analizados que, sin embargo, no desaparecen sino que son reutilizados y resignificados, jugando nuevos papeles dentro de la maraña de relaciones que conforman un paisaje. Un paisaje que, al final, resulta de una acumulación de tiempos que, entremezclados, conforman un presente complicado y complejo que puede ser una pequeña ventana para acercarnos a las personas que vivieron en él, a cómo lo pensaron, experimentaron y percibieron.

\section{Nota}

1. La presente comunicación es parte de la transferencia de conocimientos del proyecto de investigación "Vivir entre islas: paisajes insulares, conectividad y cultura material en las comunidades de las Islas Baleares durante la prehistoria reciente (2500-123 BC)." (HAR 2012 32602) financiado por el Ministerio de Economía y Competitividad.

\section{Agradecimientos}

El artículo que aquí se presenta parte de un trabajo de final de máster realizado en la Universidad Complutense de Madrid. Por ello, quiero agradecer a las tutoras de este trabajo, Marisa Ruiz-Gálvez y Carmen Mínguez sus ánimos, su ayuda y su gran paciencia. También agradecer al equipo de la Universidad de las Islas Baleares por la información aportada, así como por la catalogación de los yacimientos (especialmente a Daniel Albero, Maria Calderón y Manuel Calvo). Especialmente, agradecer a Manuel Calvo su guía, a David Javaloyas sus infinitas correcciones y a Daniel Albero sus comentarios. Mención aparte merecen todas 
las ayudas y consejos que he recibido con el SIG, especialmente gracias a Jose Señorán, Antonio Uriarte y $\mathrm{M}^{\mathrm{a}}$ Concepción Alba. Asimismo, gracias a los dos revisores del artículo por sus comentarios. Finalmente, gracias a todos los que habéis estado ahí. Cualquier error es solo mío.

\section{REFERENCIAS BIBLIOGRÁFICAS}

Albero Santacreu, D. (2011): Caracterización tecnológica, social y adaptación funcional de cerámicas prehistóricas en el oeste y Sureste de Mallorca (1700-50 BC). Aproximación sincrónica y diacrónica a partir del estudio arqueométrico de pastas. Tesis doctoral, Universidad de Granada.

Albero Santacreu, D.; Calderón Díaz, M.; Calvo Trias, M.; Gloaguen Murias, E. (2011a): Patrimonio cultural del municipio de Calvià. En Calvo y Aguareles (ed.) 2011:257-405.

Albero Santacreu, D.; García Rosselló, J.; Javaloyas Molina, D.; Calvo Trias, M. (2011b): Cultura material, habitus, espacio y movilidad en el archipiélago balear durante el Bronce final I (c.1400-1100 BC). $B S A L, 67: 15-37$.

Albero, D. (2006): Arqueología espacial en los valles de Galatzó y Es Capdellá (Mallorca). Mayurqa, $31: 137-164$.

Albero, D.; Calderón, M.; García, E.; Gloaguen, E.; Calvo, M. (2013): El navetiformes Alemany 40 años después: puesta en valor y nuevas propuestas interpretativas. BSAL, 69:15-41.

Alesan, A.; Malgosa, A. (2005): Les inhumacions perinatals del túmul de Son Ferrer (Calviá, Mallorca): Un estudi antropológic. Mayurqa, 30: 511-522.

Antrop, M. (2005): Why landscapes of the past are important for the future. Landscape and Urban Planning, 70:21-34.

Aramburu Zabala - Higuera, F. J. (1998): El patrón de asentamiento de la cultura talayótica de Mallorca. El Tall, Palma.

Aramburu, F. J.; López Quesada, Mº (1996): Los talayots cuadrados de Mallorca. Quadernos de Prehistoria y Arqueologia Casrrense, 17:183-196.

GutiérRez, J.; Ruiz-Gálvez, M.; Basildo, R.M.; (2005): Generación de un Sistema de Información Geográfica. Territorio Nurágico y Paisaje Antiguo. La meseta de Pranemuru (Cerdeña) en la Edad de Bronce (M. Ruiz-Gálvez, Ed.). Anejos de Complutum, 10:133-168.

Bradley, R. (1993): Altering the Earth: the Origins of Monuments in Britain and Continental Europe. (Monograph Series 8). Society of Antiquaries of Scotland: Edinburgh.

Calvo Trias, M.; García Rosselló, J.; Javaloyas Molina, D.; Albero Santacreu, D.; Van Strydonck, M. (2014): Matching data: analyzing the chronological use sequence in the Iron Age necropolis of the staggered turriforme of Son Ferrer (Balearic Islands, Spain). Radiocarbon, 56 (2): 361-374.

Calvo, M. (2002): El Parc Arqueologic del Puig de Sa Morisca. Universitat de les Illes Balears, Palma de Mallorca.

Calvo, M. (2009): Reflexiones en torno a los esquemas de racionalidad espacial reflejados en el paisaje durante la prehistoria de Mallorca. Pyrennae 40 (2): 37-78.

Calvo, M.; Aguareles, A. (Ed.) (2011): Calvià. Patrimonio Cultural. Vol. 1. Ajuntament de Calvià.

Calvo, M.; Fornés, J.; García, J.; Iglesias, M.A.; Juncosa, E. (2005): Condicionantes espaciales en la construcción del Turriforme escalonado de Son Ferrer (Calviá, Mallorca). Mayurqa, 30: 487-510.

Calvo, M.; Guerrero, V. (2011a): De los primeros indicios de ocupación humana en las Baleares hasta la Edad del Bronce. En Calvo y Aguareles (ed.) 2011:39-62.

Calvo, M.; Guerrero, V. (2011b): La cultura Postalayótica (650/550-123 AC). En Calvo y Aguareles (ed.) 2011:39-62.

Calvo, M.; Guerrero, V. (2011c): La cultura Talayótica. En Calvo y Aguareles (ed.) 2011:39-62.

Calvo, M.; Javaloyas, D.; Albero, D.; García Rosselló, J.; Guerrero, V. (2011): The ways people move: mobility and seascapes in the Balearic Islands during the late Bronze Age (c. 1400-850/800 bc). World Archaeology, 43 (3):345-363. 
CAlvo, M.; SAlvè, B. (1999): Aproximació a la seqüència estratigráfica y cronocultural de la naveta 1 del jaciment de Clossos de Can Gaià. Mayurqa, 25: 59-82.

CAmps, J., Vallespir, A. (1998): Excavacions a Santa Ponsa, Calvià, Mallorca: El turó de les Abeies. Consell de Mallorca, La Deixa 1, Palma.

Costa Ribas, B.; Domínguez Monedero, A.; Guerrero Ayuso, V. (2004): "Indígenes i Colons en la protohistòria tardana de les Illes". Història de les Balears. Vol. I: De la prehistòria i l'antiguitat al món islàmic (E. Berenguer, Ed.). Edicions 62, Barcelona: 188-295.

Criado Boado, F. (1989): Megalitos, espacio, pensamiento. Trabajos de Prehistoria, 46:75-98.

Criado Boado, F. (1991): Tiempos megalíticos y espacios modernos. Historia y Crítica, I: 85-108.

Criado Boado, F. (1993): Límites y posibilidades de la Arqueología del Paisaje. SPAL, Revista de Prehistoria y Arqueología, 2:9-55.

Criado Boado, F. (2012): Arqueológicas. La razón perdida. La construcción de la intel-ligència arqueològica. Bellaterra Arqueologia, Barcelona.

EnSENyAt, C. (1971): Excavaciones en el navetiforme Alemany. Noticiario Arqueológico Hispánico, 15: 9-73.

Esteban, A.; Muro, I.; Orfila Pons, M.; Vallespir, A. (1991): La reconstrucción histórica de un territorio: la bahía de Santa Ponça (Mallorca). IInd Deya International Conference of Prehistory. Recent Developments in Western Mediterranean Prehistory: Archaeological Techniques, Technology and Theory. Vol.I: Archaeological Techniques and Technology (W. H. Waldren, J. A. Ensenyat, R. C. Kennard, Eds.). BAR International Series, 573: 223-238.

FÁbrega Álvarez, P. (2006): Moving without destination. A theoretical, GIS - based determination of routes (optima accumulation model of movement from a given origin). Archaeological Computing Newsletter, 64:7-11.

FÁbrega Álvarez, P.; Parcero Oubiña, C. (2007): Proposals for an archaeological analysis of pathways and movement. Archeologia e Calcolatori, 18: 121-140.

Fornés, J.; Javaloyas, D.; Salvà, B.; Belenguer, C.; Mates, F.; Servera, G.; Oliver, L. (2007): Más que una casa. Los navetiformes de la Edad del Bronce Balear. L'espai domèstic i l'organització de la societat a la protohistòria de la Mediterrània occidental (1er millenni aC): actes de la IV Reunió internacional d'Arqueologia de Calafell: 323-330.

Garcia Amengual, E. (2014): Proposta de cadena operativa de construcció dels navetiformes del Bronze balear y la seva aplicació pràctica. DEA, Universitat de les Illes Balears.

García Rosselló, J. (2010): Análisis traceológico de la cerámica. Modelado y espacio social durante el Postalayótico (s. V-I AC) en la península de Santa Ponça (Calvià, Mallorca). Tesis doctoral UIB.

Garcia Rosselló, J.; Fornés Bisquerra, J.; Hoskin, M. (2000): Orientations of the talayotic sanctuaries of Mallorca. Archaeoastronomy, 25:58-76.

Garcia Rosselló, J.; Quintana, C. (2003): Cerámica indígena y cerámica a torno: una aportación a la producción cerámica talayótica tardía de Mallorca. Mayurqa, 29:281-300.

Garcias Mas, P.; Gloaguen, E. (2004): Los enterramientos infantiles en el túmulo de Son Ferrer (Calviá, Mallorca): Una primera aproximación. Mayurqa, 29: 269-280.

GiLI, S. (1995): Territorialidades de la prehistoria reciente mallorquina. Tesis doctoral, Universidad Autónoma de Barcelona.

González Ruibal, A. (2006): The Past is Tomorrow: Towards an Archaeology of the Vanishing Present. Norwegian Archaeological Review, 39 (2): 110-125.

Grimalt Vigo, F. (2001): Els camins de Calvià. Premi Rei En Jaume d'Investigació. Ajuntament de Calvià, Mallorca.

Guerrero, V. (1982): Los núcleos arqueológicos de Calviá. Ajuntament de Calviá, Mallorca.

Guerrero, V. (1997): Colonización púnica de Mallorca. La documentación arqueológica y el context histórico. El Tall, Palma de Mallorca.

Guerrero, V. (1999): Arquitectura y poder en la prehistoria de Mallorca. El Tall, Palma de Mallorca.

Guerrero, V. (2001): The Balearic Islands: Prehistoric Colonization of the Furthest Mediterranean Islands from the Mainland. Journal of Mediterranean Archaeology 14 (2): 136-158. 
Guerrero, V.; Calvo, M.: Gornés, S. J. (2006a): El poblamiento prehistórico de las islas Baleares. Historia de las Baleares Vol. I, Ed. Rey Sol, Palma.

Guerrero, V.; Calvo, M.: Gornés, S. J. (2006b): Mallorca y Menorca en la Edad del Hierro. Historia de las Baleares Vol. II, Ed. Rey Sol.

Guerrero, V.; Calvo, M.; García, J.; Gornés, S. (2007): Prehistoria de las Islas Baleares: Registro arqueológico y evolución social antes de la Edad del Hierro. B.A.R. International Series 1690. Archaeopress, Oxford.

Guerrero, V.; Calvo, M.; Salvì, B. (2002): La Cultura Talayótica: Una sociedad de la Edad del Hierro en la periferia de la colonización púnica. Complutum, 13: 221-258.

HeRnÁNDEZ Gasch, J.; Quintana, C. (2013): Cuando el vino impregnó la isla de Mallorca: el comercio púnico - ebusitano y las comuniddes locales durante la segunda mitad del siglo V y el siglo IV a.C. Trabajos de Prehistoria, 70 (2):315-331.

HeRnÁndez Gasch, J. (1998): Las necrópolis de la Edad del Hierro de Son Real y s'Illa des Porros (Santa Margalida, Mallorca). Estudio arqueológico y análisis social. Arqueomediterrània 3, Universitat de Barcelona, Barcelona.

Hernández, M. A.; García, E.; Salva, B.; Fornés, J. (2003): L'àrea domèstica de la Unitat Estratigràfica 9 de la Naveta I dels Closos de can Gaià. II Jornades d'Estudis Locals de Felanitx: 137-161.

INGOLD, T. (1993): The temporality of the landscape. World Archaeology, 25 (2):152-174.

Ingold, T. (2011): Being Alive: essays on movement, knowledge and description. Routledge, Londres y Nueva York.

Javaloyas, D.; Fornés, J.; Salvà, B.; Oliver, L.; Servera, G. (2011): Concepcions espacials a l'Edat del Bronze. Els navetiformes: entre l'espai public i el privat. III Jornades d'Arqueologia de les Illes Balears: 23-34.

Llobera, M. (2003): Extending GIS - based visual analysis: the concept of visualscapes. International Journal of Geographical Information Science, 17 (1): 25-48.

Lull, V.; Micó, R.; Rinuete, C.; Risch, R. (2001): La prehistoria de las Islas Baleares y el yacimiento arqueológico de Son Fornés (Montuiri, Mallorca). Fundación Son Fornés, Barcelona.

Parcero OubiÑa, C. (2000): La construcción del paisaje social en la Edad del Hierro del Noroeste Ibérico. Tesis doctoral, Universidad de Santiago de Compostela.

Parcero Oubiña, C. (2005): Variaciones en la función y el sentido de la fortificación a lo largo de la Edad del Hierro en el NO de la Península Ibérica. Bronce Final y Edad del Hierro en la Península Ibérica. Actas del Encuentro de Jóvenes Investigadores (A. Blanco; C. Cancelo; A. Esparza, Eds). Colección Aquilafuente, Vol. 83, Salamanca: 11-33.

Parcero Oubiña, C.; Fábrega Álvarez, P.; Güimil Fariña, A.; Fonte, J.; Valdez, J. (2009): Castros, caminos, rutas y ocupación del espacio. Modelización y análisis de las formas de movilidad asociadas a los asentamientos de la Edad del Hierro a través de herramientas SIG. Arte rupestre, paleoambiente y paisaje. Miradas interdisciplinares sobre Campo Lameiro (F. Criado Boado; A. Martínez Cortizas, Eds.). Col. TAPA, CSIC, Santiago de Compostela.

Picornell Gelabert, Ll. (2012): Paisaje vegetal y comunidades prehistóricas y protohistóricas en Mallorca y Menorca (Illes Balears): una aproximación desde la antracología. Tesis doctoral, Universitat de Barcelona.

Pons Homar, G. (1999): Anàlisi espacial del poblament al Pretalaiòtic final i Talaiòtic I de Mallorca (ss. $X I X-V I I$ A. C.). Col·lecció La Deixa. Monografies de Patrimoni Històric. Consell de Mallorca, Palma.

Quintana, C. (2000): La ceràmica superficial d'importació del Puig de Sa Morisca. Valldargent, Ajuntament de Calvià, Palma.

Richards, C. (2000): Monumental choreography. Architecture and Spatial Representation in Late Neolithic Orkney. Interpretative Archaeology. A reader (J. Thomas, Ed.). Leicester University Press, Londres y Nueva York.

Rinuete HerRada, C. (2000): Dimensiones bio - arqueológicas de los contextos funerarios. Estudio de los restos humanos de la necròpolis prehistórica de la Cova des Càrritx (Ciutadella, Menorca). Tesis doctoral, Universitat Autònoma de Barcelona. 
Rodríguez Alcalde, A. L. (1995): Aproximación estadística al paisaje humano en la Prehistoria de Mallorca. Complutum, 6:167-192.

Salvà, B. (2001): El Pretalaiòtic al llevant mallorquí (1700-1000 AC). Anàlisi Territorial. Edicions Documenta Balear, Palma.

Salvà, B.; CAlvo, M.; Guerrero, V. (2002): La Edad del Bronce balear (1700-1000/900 BC): Desarrollo de la complejidad social. Complutum, 13: 193-219.

SALVÀ, B.; HeRnÁndEZ, J. (2009): Los espacios domésticos en las Islas Baleares durante las edades del bronce y del hierro. De la sociedad naviforme a la talayótica. L'espai domèstic i l'organització de la societat a la protohistòria de la Mediterrània occidental (Ier mil.leni a.C.) ( M. C. Belarte, Ed.). Arqueomediterrània 11, Calafell, 6-9 marzo de 2007.

SANTOS GRANERo, F (2004): Escribiendo la historia en el paisaje: espacio, mitología y ritual entre la gente yanesha. En Surrallés, A.; García Hierro, P. (Ed.) (2004): Tierra Adentro. Territorio indígena y percepción del entorno. IWGIA (Grupo Internacional de Trabajo sobre Asuntos Indígenas), Lima.

Schlanger, S. H. (1992): Recognizing Persistent Places in Anasazi Settlement Systems. Space, Time and Archaeological Landscapes (J. Rossignol, L. Wandsnider, eds.), Springer, Nueva York.

SHANG, H.; Bishop, I.D. (2000): Visual tresholds for detection, recognition and visual impact in landscape settings. Journal of Environmental Psychology, 20:125-140.

Tilley, C. (1994): Phenomenology of Landscape. Places, Paths and Monuments. Berg, Oxford / Providence, USA.

Tilley, C. (2004). Mind and Body in Landscape Research. Cambridge Archaeological Journal, 14 (1): $77-$ 80.

TobleR, W. (1993): Three presentations on geographical analysis and modelling. Santa Barbara: National Center for Geographic Information and Analysis. [URL: http://www.ncgia.ucsb.edu/Publications/Tech_ Reports/93/93-1.PDF.] Acceso el 15/05/2014.

Trigger, B. (1990): Monumental Architecture: A Thermodynamic Explanation of Symbolic Behaviour. World Archaeology, 22 (2): 119-132.

Vives Reus, M. (2011): Calvià en la época contemporània. En Calvo y Aguareles (Ed.) 2011: 221-256.

Weathley, D. (1995): Cumulative Viewshed Analysis: a GIS - based method for investigating intervisibility, and its archaeological application. Archaeology and GIS: a European Perspective (G. Lock, Z. Stancic, Eds.). Routledge, Londres. 